\title{
Clinical Study \\ Electromyographic Activity in the EEG in Alzheimer's Disease: Noise or Signal?
}

\author{
Karin van der Hiele, ${ }^{1,2}$ Robert H. A. M. Reijntjes, ${ }^{3}$ Alla A. Vein, ${ }^{3}$ \\ Rudi G. J. Westendorp, ${ }^{4}$ Mark A. van Buchem, ${ }^{5}$ Eduard L. E. M. Bollen, ${ }^{6}$ \\ Huub A. M. Middelkoop, ${ }^{1,2}$ and J. Gert van Dijk ${ }^{3}$ \\ ${ }^{1}$ Neuropsychology, Department of Neurology, Leiden University Medical Centre, P.O. Box 9600, 2300 RC Leiden, The Netherlands \\ ${ }^{2}$ Department of Psychology, Clinical, Health and Neuropsychology, Faculty of Social Sciences, Leiden University, P.O. Box 9555, \\ 2300 RB Leiden, The Netherlands \\ ${ }^{3}$ Clinical Neurophysiology, Department of Neurology, Leiden University Medical Centre, P.O. Box 9600, 2300 RC Leiden, \\ The Netherlands \\ ${ }^{4}$ Department of Gerontology and Geriatrics, Leiden University Medical Centre, P.O. Box 9600, 2300 RC Leiden, The Netherlands \\ ${ }^{5}$ Department of Radiology, Leiden University Medical Centre, P.O. Box 9600, 2300 RC Leiden, The Netherlands \\ ${ }^{6}$ Department of Neurology, Leiden University Medical Centre, P.O. Box 9600, 2300 RC Leiden, The Netherlands
}

Correspondence should be addressed to Karin van der Hiele, hiele@fsw.leidenuniv.nl

Received 8 November 2010; Accepted 26 January 2011

Academic Editor: Sara Määttä

Copyright (๑) 2011 Karin van der Hiele et al. This is an open access article distributed under the Creative Commons Attribution License, which permits unrestricted use, distribution, and reproduction in any medium, provided the original work is properly cited.

\begin{abstract}
Many efforts have been directed at negating the influence of electromyographic (EMG) activity on the EEG, especially in elderly demented patients. We wondered whether these "artifacts" might reflect cognitive and behavioural aspects of dementia. In this pilot study, 11 patients with probable Alzheimer's disease (AD), 13 with amnestic mild cognitive impairment (MCI) and 13 controls underwent EEG registration. As EMG measures, we used frontal and temporal 50-70 Hz activity. We found that the EEGs of AD patients displayed more theta activity, less alpha reactivity, and more frontal EMG than controls. Interestingly, increased EMG activity indicated more cognitive impairment and more depressive complaints. EEG variables on the whole distinguished better between groups than EMG variables, but an EMG variable was best for the distinction between MCI and controls. Our results suggest that EMG activity in the EEG could be more than noise; it differs systematically between groups and may reflect different cerebral functions than the EEG.
\end{abstract}

\section{Introduction}

There is at present no certain diagnostic test for Alzheimer's disease $(\mathrm{AD})$. Much attention is devoted to identify specific markers for $\mathrm{AD}$ and its presumed precursor stage, amnestic mild cognitive impairment (MCI). One research technique concerns the electroencephalogram (EEG), which has the advantages of easy availability and low cost.

Research EEG efforts focused on the search for parameters that may help distinguish between healthy ageing, MCI, and $\mathrm{AD} . \mathrm{AD}$ is characterised by slowing of the EEG, which finding can be quantified using spectral analysis [1]. Resulting variables such as ratios between fast and slow activity, alpha and theta relative power, or the mean frequency have shown varying degrees of efficacy in this respect $[2,3]$. Attempts have been made to increase the differences between groups by challenging the subjects, such as by a memory task or more simply by comparing "eyes open" with "eyes closed" conditions. For example, studies comparing AD with healthy controls reported increased theta power and decreased alpha suppression during eye opening and a memory task $[1,2$, 4-6]. Compared to controls, EEG abnormalities in MCI consisted of decreased alpha suppression and increased alpha power during a memory task $[7,8]$. Such reports suggest that the EEG may have an ancillary role in the diagnostic workup of $\mathrm{AD}$ and MCI, but it has not achieved a prominent place in 
the diagnostic arsenal [9]. Many attempts have been made to improve the yield of the EEG for this purpose. It is commonly considered of the utmost importance to obtain a high-quality EEG, uncontaminated by artifacts such as blinks, eye movements, and electromyographic (EMG) activity, which can be extremely difficult in dementia. Eye blinks and movements most often cause low frequency potentials, and may therefore affect the measurement of low frequency signals in the EEG, that is, delta and theta waves $[10,11]$. EMG activity is usually due to activity of scalp, facial, and jaw muscles and typically most strongly affects frontal and temporal EEG recordings [12]. It mostly affects the high-frequency gamma and beta bands. Over the years researchers devoted much attention to dealing with these unwanted influences. One approach is to reject contaminated epochs from analysis, either manually or through automated methods $[2,10,13]$. Others attempted to negate effects of artifacts using various techniques [14-18], and yet others chose to simply disregard affected frequency bands, largely leaving theta and alpha activity [8]. The rationale behind these intensive efforts to remove eye blinks, movements, and EMG from the EEG is probably that the latter reflects "pure" cerebral activity, whereas the "artifacts" do not. We do not doubt that the EEG reflects cerebral activity, but wondered whether the other group of parameters can be dismissed out of hand. They may be regarded as behavioural correlates, and AD ultimately affects behaviour, in a chain of events involving amongst others biochemistry, neuronal function, and cognition. It is conceivable that the amount of muscle activity is related to the degree of cognitive impairment or to neuropsychiatric symptoms such as agitation, irritability, and depression. If so, then these behavioural correlates may reflect the presence of AD. How well the EEG does so has been investigated, but how well behavioural "contaminations" do so has, to our knowledge, not been tested.

In this pilot study, we explored the discriminative value of quantitative EEG and EMG in distinguishing between $\mathrm{AD}$, MCI and healthy aging. Furthermore, we examined whether EMG activity was related to neuropsychiatric and cognitive measures.

\section{Materials and Methods}

2.1. Participants. Thirteen age-matched controls, 13 patients diagnosed with MCI [19], and 11 diagnosed with probable $\mathrm{AD}[20]$ participated in the study. Patients had been referred to the outpatient memory clinic of the Leiden University Medical Centre. Control subjects without cognitive complaints were recruited through an advertisement in a local newspaper. All patients and controls underwent general medical, neurological, neuropsychological, and brain MRI investigations as part of the standardized dementia screening. Furthermore, all patient histories were reviewed and diagnoses reached in multidisciplinary consensus meetings. Within three months from the standardized dementia screening, patients and controls participated in an additional EEG examination. Eligible subjects had to be free of psychotropic medication, aged 60 years or above, and without previous history of psychiatric and neurological disorders or substance abuse. Moreover, they had no abnormalities on MRI other than white matter hyper intensities or an incidental small lacunar lesion ( $\leq 5 \mathrm{~mm}$ diameter). The study was approved by the local Medical Ethical Committee. Written informed consent was obtained from all subjects, or from close relatives or caregivers in case of dementia.

2.2. Neuropsychiatric and Cognitive Assessment. The Geriatric Depression Scale (GDS), a patient-based interview, was used to determine the presence and severity of depressive feelings [21]. We used the Neuropsychiatric Inventory (NPI-Q) [22], an informant-based interview, to determine the presence and severity of neuropsychiatric problems (i.e., delusions, hallucinations, agitation, depression, anxiety, euphoria, apathy, disinhibition, irritability, aberrant motor activity, night-time behavioural disturbances, and eating disturbances). Per item a score from " 0 " to " 3 " could be obtained, with " 0 " indicating the absence of a neuropsychiatric problem and "1", "2", and " 3 " indicating a mild, moderate, and severe neuropsychiatric problem, respectively. NPI items were clustered based on previous research using principal component analysis [23]. Three factors were used (1) a "mood/apathy" factor including depression, apathy, night-time behavioural disturbances, and eating disturbances, (2) a "hyperactivity factor" including agitation, euphoria, irritability, disinhibition, and aberrant motor activity, and (3) a "psychosis" factor including hallucinations and delusions. Anxiety was considered a separate factor, but it was excluded from further analysis because of limited variability in scores. The Cambridge cognitive examination (CAMCOG) [24] was used to provide a measure of general cognitive functioning.

2.3. EEG Recording and Analysis. EEGs were recorded using a Nihon Kohden 2110 apparatus with $21 \mathrm{Ag} / \mathrm{AgCl}$ electrodes placed according to the 10/20 system. ECG, respiration, and horizontal eye movement leads were recorded to facilitate recognition of artifacts. The EEG was band-pass filtered from $0.16-70 \mathrm{~Hz}$ for display and analysis but recorded unfiltered. The sample frequency was $200 \mathrm{~Hz}$ and the $\mathrm{AD}$ precision 12 bits. The average reference montage was used, with the exclusion of electrodes Fp1, Fp2, A1, and A2. All EEGs were recorded in the afternoon. During recording, subjects sat slightly reclined in a comfortable chair, approximately $1.5 \mathrm{~m}$ in front of a computer screen. The light in the room was dimmed.

The EEG was registered during a 10-minute eyes-closed period, a 3-minute eyes-open period, and during memory activation. The memory activation task concerned picture memory. Subjects were consecutively shown 10 pictures of common objects on a computer screen. Each picture was presented for two seconds and subjects were asked to name the shown objects aloud. After presentation of the pictures, subjects had to close their eyes and memorise the pictures for 15 seconds. This period was later used for data analysis. Subjects were then asked to open their eyes and name as many pictures they could remember. The task was performed three times using the same 10 pictures. The number of remembered pictures was noted. 
2.4. EEG Parameters. To study EEG parameters, we selected specific periods of the EEG using visual analysis. Samples had to be free of eye movements, blinks, and muscle activity. Sample selection was performed by the first author and corroborated by an experienced clinical neurophysiologist. Both were blind to clinical diagnosis. Samples had to be $4-8$ seconds in length and were selected during conventional eyes closed and eyes open, and memory activation. Frequency analysis was performed using a fast Fourier transformation. We calculated absolute power in theta $(4-8 \mathrm{~Hz})$ and alpha $(8-13 \mathrm{~Hz})$ frequency bands.

Three parameters were then calculated: theta relative power during eyes closed, and alpha reactivity during both eye opening and memory activation. Alpha reactivity is defined as the percentile decrease in absolute alpha power as compared to the eyes closed condition. EEG parameters were averaged over all electrodes and over the three selected EEG samples available for each of the eyes closed, eyes open, and memory activation periods. We chose these EEG parameters as they were found sensitive to $\mathrm{AD}$ and $\mathrm{MCI}$ in previous research $[1,2,4-6,8]$. All signal processing was performed using MATLAB (The MathWorks, Natick, USA).

2.5. EMG Parameters. To measure the amount of EMG activity, we did not select specific periods but used the entire period corresponding to the eyes closed, eyes open, and each of the three memory activation periods. The only selection involved was that periods in which the tasks were compromised by matters such as electrode correction were omitted. Frequency analysis was performed using a fast Fourier transformation. As a measure of EMG activity, absolute power was calculated in the high frequency band $(50-70 \mathrm{~Hz})$ and averaged over temporal (T3, T4, T5, and T6) and frontal electrodes (F3, F4, F7, and F8). These sites are sensitive to EMG activity from scalp, facial, and jaw muscles. The $50 \mathrm{~Hz}$ threshold was chosen to exclude any possible confusion with brain activity, that is, beta or gamma activity, that might be present at lower frequencies. In doing so, we aimed to ensure that the measured activity was the result of muscle activity only.

For analysis, we used temporal and frontal EMG during eyes closed, eyes open, and memory activation. EMG parameters were averaged over the three samples available for the memory activation periods.

2.6. Statistical Analysis. SPSS for Windows (release 14.0.1) was used for data analysis. Group differences in cognitive and neuropsychiatric outcomes, EEG, and EMG activity were assessed using ANOVA with post hoc Bonferroni tests. When data were not normally distributed we used twosample Kolmogorov-Smirnov tests to assess group differences. Subsequently, we performed a two-tailed parametric or nonparametric correlation analysis to investigate relations between EEG and EMG activity on the one hand and cognitive and neuropsychiatric parameters on the other. We did not investigate correlations in specific diagnostic groups because of the limited number of subjects per group. The level of significance was set at $P \leq .05$.
TABLE 1: Clinical characteristics.

\begin{tabular}{lccc}
\hline & Controls & MCI & AD \\
\hline male/ female $^{\mathrm{a}}$ & $3 / 10$ & $6 / 7$ & $6 / 5$ \\
age (years) & $73(5)$ & $73(5)$ & $75(8)$ \\
$\begin{array}{l}\text { education (years) } \\
\text { CAMCOG (max 106) }\end{array}$ & $10(3)$ & $11(4)$ & $10(4)$ \\
$\begin{array}{l}\text { Picture memory score } \\
\text { (max 30) }\end{array}$ & $25(2)$ & $15(3)^{* *}$ & $10(4)^{* * \# \#}$ \\
$\begin{array}{l}\text { GDS (max 15) } \\
\text { NPI “mood/apathy" }\end{array}$ & $0.8(1.2)$ & $2.1(1.7)$ & $1.8(1.6)$ \\
$\begin{array}{l}\text { (max 12) } \\
\text { NPI "hyperactivity" } \\
\text { (max 15) }\end{array}$ & - & $2.0(1.2)$ & $2.4(2.7)$ \\
$\begin{array}{l}\text { NPI “psychosis" } \\
\text { (max 6) }\end{array}$ & - & $2.0(1.9)$ & $2.8(1.9)$ \\
\hline
\end{tabular}

Values in the table are means with S.D. in parentheses. ANOVA was used to assess group differences, except for: ${ }^{\mathrm{a}} \chi^{2}$-test was used and ${ }^{\mathrm{b}}$ Nonparametric two sample Kolmogorov-Smirnov tests were used. In view of the small number of MCI and AD patients for whom an NPI was available, we did not

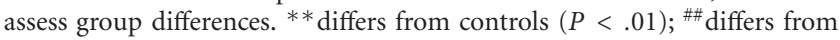
MCI patients $(P<.01)$. CAMCOG: Cambridge Cognitive Examination; GDS: Geriatric Depression Scale (N: 13 controls, 12 MCI and 9 AD); NPI: Neuropsychiatric Inventory (N: $5 \mathrm{MCI}$ and $5 \mathrm{AD}$ ).

The aim of the study was to compare not only whether EEG and EMG parameters differed between groups but also whether they did so to different degrees. We assumed they could not be compared directly, because of wholly different distribution patterns including various nonnormal distributions. We also wished to avoid subjective choices regarding abnormality thresholds. To avoid such problems, we chose to plot receiver operating curves. The area under the curve serves as a quantitative indicator of how well a specific parameter distinguishes between groups, irrespective of the nature of the parameter in question. This value usually ranges between 0.5 (meaning the parameter does not distinguish between the groups at all) to 1.0 (the parameter separates the two groups perfectly). We will assume that values below 0.75 have little relevance.

\section{Results}

3.1. Clinical Characteristics. Clinical characteristics are shown in Table 1. Sex, age, years of education, and GDS scores did not differ between groups. Group differences were found in CAMCOG scores $(F(2,34)=50.0 ; P<.001)$. Post hoc tests showed that AD patients had significantly lower CAMCOG scores than controls and MCI patients $(P<.001)$. Furthermore, MCI patients had lower CAMCOG scores $(P<.001)$ than controls. There were also differences in the number of pictures correctly remembered in the memory task used during EEG registration $(F(2,34)=72.6$; $P<.001)$. Post hoc tests showed that $\mathrm{AD}$ and MCI patients remembered less pictures than controls $(P<.001)$. Furthermore, $\mathrm{AD}$ patients had a lower score than MCI patients $(P<.01)$. 
3.2. EEG and EMG. Table 2 shows EEG and EMG data. Group differences in theta relative power were found during eyes closed $(F(2,34)=10.6 ; P<.001)$. Post hoc tests indicated that $\mathrm{AD}$ patients showed significantly higher theta relative power as compared with controls and MCI patients (both $P<.001$ ). Alpha reactivity upon eyes opening and memory activation also differed between groups $(F(2,34)=$ 5.3; $P<.01$ and $(F(2,34)=6.1 ; P<.01)$ in that alpha reactivity was decreased in $\mathrm{AD}$ patients as compared with controls.

Group differences in frontal EMG were found during both eyes closed and eyes open $(F(2,34)=3.8 ; P<.05$ and Kolmogorov-Smirnov $Z=1.4 ; P<.05)$. AD patients showed more frontal EMG activity than controls.

3.3. Correlation Analyses. Correlations are shown in Table 3. Theta relative power, alpha reactivity during eyes open, and alpha reactivity during memory activation were related to CAMCOG scores; lower theta relative power and higher alpha reactivity indicated higher CAMCOG scores, and thus better cognitive performance. Furthermore, frontal EMG during eyes open was related to CAMCOG scores in that increased EMG activity indicated lower CAMCOG scores.

Correlations were found between, on the one hand, frontal EMG during eyes closed, temporal EMG during eyes closed, and temporal EMG during eyes open and, on the other hand, GDS scores (see Table 3 ). In all cases, increased EMG activity indicated more complaints of depression. No correlations were found between EEG and EMG parameters and neuropsychiatric complaints on the NPI.

3.4. Receiver Operating Curves. ROC data are shown in Table 4. Of the 9 EEG ROCs, 4 reached levels above 0.75 . Three of these concerned the comparison between $\mathrm{AD}$ patients and controls, and the fourth the $\mathrm{AD}-\mathrm{MCI}$ comparison.

Of the 18 EMG ROCs, 3 reached levels above 0.75 . Two concerned the $\mathrm{AD}$-control comparison, and one the $\mathrm{MCI}-$ control comparison.

\section{Discussion}

EMG activity is usually regarded a source of problems in EEGs used for patient care and research purposes in dementia, because the EMG impairs the registration of the EEG. This is particularly relevant for activity at the high (beta and gamma) and low (delta) ends of the spectrum. The most important new finding of this pilot study is that EMG activity could be not only a hindrance but also reflects some aspects of $\mathrm{AD}$ and MCI. We base this conclusion on two lines of evidence.

Firstly, the amount of frontal EMG activity was higher in the $\mathrm{AD}$ group than in controls, both during the eyes open and eyes closed conditions. As such, its presence is linked to the presence of $\mathrm{AD}$, presumably through an association between facial muscle activity and the expression of emotions such as depression. The ROC analysis corroborates this finding. We used this type of analysis to allow us to compare variables of
TABLE 2: EEG and EMG data.

\begin{tabular}{lccc}
\hline & Controls & MCI & AD \\
\hline Eyes closed & & & \\
\hline EEG theta relative power $(\%)$ & $28(8)$ & $29(14)$ & 53 \\
& $21)^{* * \#}$ \\
Frontal EMG $\left(\mu \mathrm{V}^{2}\right)$ & $2(1)$ & $6(5)$ & $7(7)^{*}$ \\
Temporal EMG $\left(\mu \mathrm{V}^{2}\right)$ & $4(4)$ & $9(8)$ & $8(10)$ \\
\hline Eyes open & & & \\
\hline EEG alpha reactivity $(\%)$ & $67(20)$ & $59(18)$ & $37(32)^{* *}$ \\
Frontal EMG $\left(\mu \mathrm{V}^{2}\right)^{\mathrm{a}}$ & $6(4)$ & $16(25)$ & $24(34)^{*}$ \\
Temporal EMG $\left(\mu \mathrm{V}^{2}\right)^{\mathrm{a}}$ & $10(8)$ & $15(25)$ & $18(30)$ \\
\hline Memory activation & & & \\
\hline EEG alpha reactivity $(\%)$ & $40(33)$ & $10(32)$ & $-7(35)^{* *}$ \\
Frontal EMG $\left(\mu \mathrm{V}^{2}\right)^{\mathrm{a}}$ & $3(2)$ & $13(15)$ & $21(41)$ \\
Temporal EMG $\left(\mu \mathrm{V}^{2}\right)^{\mathrm{a}}$ & $6(5)$ & $19(26)$ & $14(16)$ \\
\hline
\end{tabular}

Values in the table are means with S.D. in parentheses. ANOVA was

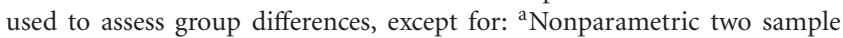
Kolmogorov-Smirnov tests were used. ${ }^{*}$ differs from controls $(P \leq .05)$; ** differs from controls $(P \leq .01)$; ${ }^{\#}$ differs from MCI patients $(P<.01)$.

a different nature, that is, EEG and EMG. This comparison shows that EEG variables on the whole distinguish better between the groups than EMG variables and also that EMG variables are not worthless in this respect. In fact, for the distinction between MCI and controls, it was an EMG variable that best distinguished between groups.

Secondly, EMG activity cannot be considered as an aspecific finding: we found significant relations between EMG activity and patient-based reports of depression. It, therefore, seems likely that the EMG reflects disease-related neuropsychiatric changes. The relation between facial EMG activity and depression has been studied previously: for instance, when depressed subjects generated sad thoughts or simply thought about a typical day in their current lives, the amount of facial EMG activity increased [25]. It can be imagined that a degree of depression in patients with memory complaints is expressed as an increased facial EMG. As one might expect, different emotions lead to different patterns of EMG activity in various muscles [26]. In the context of an EEG, it seems likely that activity of the frontalis, corrugator supercilii, and zygomatic major muscles are picked up preferentially. These muscles are indeed involved in affective reactions, such as distress and pleasure. Note that we made no effort to record these muscles with any degree of precision, so we refrain from drawing detailed conclusions in this regard.

It may well be argued that even if there is more EMG activity in $\mathrm{AD}$ than in controls, this has little bearing on the understanding of the pathophysiology of $\mathrm{AD}$. In part we agree; the EMG reflects muscle activation and is thus a behavioural correlate, while the EEG is a direct reflection of cerebral function. Decreased EEG activity has been linked with functional disconnections among cortical areas, resulting among others from neuronal death and/or deficiency of neurotransmitters [1]. This is no doubt true, but it should be kept in mind that it is doubtful which cerebral functions are expressed in the various rhythms of 
TABLE 3: Correlations between EEG and EMG activity on the one hand and cognitive and neuropsychiatric scores on the other hand.

\begin{tabular}{|c|c|c|c|c|c|}
\hline & CAMCOG & GDS $^{\mathrm{a}}$ & NPI mood/apathy & NPI hyperactivity & NPI psychosis ${ }^{\mathrm{a}}$ \\
\hline \multicolumn{6}{|l|}{ Eyes closed } \\
\hline $\begin{array}{l}\text { EEG theta relative } \\
\text { power }\end{array}$ & $-0.54^{* *}$ & 0.32 & -0.45 & -0.08 & -0.14 \\
\hline Frontal EMG & -0.25 & $0.52^{* *}$ & 0.43 & 0.30 & -0.11 \\
\hline Temporal EMG & -0.12 & $0.39^{*}$ & -0.01 & -0.32 & -0.24 \\
\hline \multicolumn{6}{|l|}{ Eyes open } \\
\hline EEG alpha reactivity & $0.53^{* *}$ & -0.04 & 0.43 & 0.17 & -0.02 \\
\hline Frontal EMG ${ }^{\mathrm{a}}$ & $-0.43^{* *}$ & 0.33 & 0.13 & 0.20 & -0.01 \\
\hline Temporal EMG ${ }^{\mathrm{a}}$ & -0.05 & $0.35^{*}$ & 0.42 & 0.07 & -0.24 \\
\hline \multicolumn{6}{|l|}{ Memory activation } \\
\hline EEG alpha reactivity & $0.43^{* *}$ & -0.28 & 0.11 & 0.23 & -0.37 \\
\hline Frontal EMG ${ }^{\mathrm{a}}$ & -0.24 & 0.28 & -0.21 & 0.07 & -0.07 \\
\hline Temporal EMG ${ }^{\mathrm{a}}$ & -0.30 & 0.28 & -0.06 & -0.11 & -0.36 \\
\hline
\end{tabular}

Values in the table are Pearson's correlation coefficients. In a Spearman's correlation coefficients are displayed, as values were not normally distributed. ${ }^{*} P \leq .05$ and ${ }^{*} P \leq .01$. Significant correlations are printed in bold. CAMCOG: Cambridge Cognitive Examination; GDS: Geriatric Depression Scale (N: 13 controls, $12 \mathrm{MCI}$ and $9 \mathrm{AD}$ ); NPI: Neuropsychiatric Inventory (N: 5 MCI and $5 \mathrm{AD}$ ).

TAble 4: Classification accuracy of EEG and EMG parameters by receiver operating curves (ROC).

\begin{tabular}{lllc}
\hline & \multicolumn{3}{c}{ ROC area under the curve } \\
& $\begin{array}{c}\text { AD versus } \\
\text { controls }\end{array}$ & $\begin{array}{c}\text { MCI versus } \\
\text { controls }\end{array}$ & $\begin{array}{c}\text { AD versus } \\
\text { MCI }\end{array}$ \\
\hline Eyes closed & & & \\
\hline EEG theta relative power $(\%)$ & $\mathbf{0 . 9 2}$ & 0.50 & $\mathbf{0 . 8 5}$ \\
Frontal EMG $\left(\mu \mathrm{V}^{2}\right)$ & $\mathbf{0 . 7 8}$ & $\mathbf{0 . 8 3}$ & 0.52 \\
Temporal EMG $\left(\mu \mathrm{V}^{2}\right)$ & 0.64 & 0.73 & 0.41 \\
\hline Eyes open & & & \\
\hline EEG alpha reactivity $(\%)$ & $\mathbf{0 . 8 2}$ & 0.65 & 0.72 \\
Frontal EMG $\left(\mu \mathrm{V}^{2}\right)$ & $\mathbf{0 . 8 6}$ & 0.61 & 0.71 \\
Temporal EMG $\left(\mu \mathrm{V}^{2}\right)$ & 0.49 & 0.51 & 0.50 \\
\hline Memory activation & \multicolumn{3}{c}{} \\
\hline EEG alpha reactivity $(\%)$ & $\mathbf{0 . 8 4}$ & 0.74 & 0.67 \\
Frontal EMG $\left(\mu \mathrm{V}^{2}\right)$ & 0.74 & 0.73 & 0.47 \\
Temporal EMG $\left(\mu \mathrm{V}^{2}\right)$ & 0.69 & 0.70 & 0.49 \\
\hline Areas under the curve of & 0.75 &
\end{tabular}

Areas under the curve of over 0.75 are printed in bold.

the EEG. For example, slowing of the EEG is not specific to the type of dementia, in spite of completely different pathophysiological substrates. In fact, EEG slowing is not even specific to dementia, as this also occurs in a large variety of other conditions ranging from mechanical trauma to intoxications. One may wonder whether EEG slowing is really so much closer to the pathology of $\mathrm{AD}$ than $\mathrm{EMG}$ activity.

In the present study, the EEG generally resulted in a better discrimination between groups than the EMG, except for the MCI-control distinction, in which the EMG performed better. We do not suggest that henceforth the EMG should be used for discriminating MCI patients and controls, as we made no attempt to optimise recordings for this purpose.
For similar reasons, we did not calculate measures such as sensitivity and specificity even though many attempts have been made to optimise the EEG for this purpose. Regarding optimisation, the best parameter might be the one that accurately reflects the presence of a pathogenic cause. An example is measuring the number of CAG repeats in Huntington's disease, reflecting the pathogenetic cause. The test however does not reflect the degree of affliction at all. EEG parameters do not reflect the pathogenetic cause but the degree of affliction. As this varies from normal to severely abnormal, there must be an overlap in function in early phases. This may be seen as a failure of the test to pick up any differences, but it seems more likely that the overlap truthfully represents an overlap in function. The best we can do may be to push against the detection boundary, and in this respect, it may pay to keep an open mind regarding the nature of the parameter to be measured.

\section{Conflict of Interests}

There are no potential conflict of interests, including any financial, personal or other relationships with people or organizations that could inappropriately influence the current study. The study has been approved by the local Medical Ethical Committee.

\section{Acknowledgments}

The authors thank E. L. Tiesma, A. van der Welle, P. J. van Someren, J. G. van Vliet-de Regt, M. J. Stijl-Pek, J. C. Albusde Meza, A. J. C. van der Kamp-Huyts, F. I. Kerkhof, and P. Massaar for their help in acquiring the EEG data.

\section{References}

[1] J. Jeong, "EEG dynamics in patients with Alzheimer's disease," Clinical Neurophysiology, vol. 115, no. 7, pp. 1490-1505, 2004. 
[2] K. Bennys, G. Rondouin, C. Vergnes, and J. Touchon, "Diagnostic value of quantitative EEG in Alzheimer's disease," Neurophysiologie Clinique, vol. 31, no. 3, pp. 153-160, 2001.

[3] V. Jelic, S. E. Johansson, O. Almkvist et al., "Quantitative electroencephalography in mild cognitive impairment: longitudinal changes and possible prediction of Alzheimer's disease," Neurobiology of Aging, vol. 21, no. 4, pp. 533-540, 2000.

[4] M. Grunwald, F. Busse, A. Hensel et al., "Correlation between cortical $\theta$ activity and hippocampal volumes in health, mild cognitive impairment, and mild dementia," Journal of Clinical Neurophysiology, vol. 18, no. 2, pp. 178-184, 2001.

[5] A. Stevens, T. Kircher, M. Nickola, M. Bartels, N. Rosellen, and H. Wormstall, "Dynamic regulation of EEG power and coherence is lost early and globally in probable DAT," European Archives of Psychiatry and Clinical Neuroscience, vol. 251, no. 5, pp. 199-204, 2001.

[6] K. van der Hiele, A. A. Vein, A. van der Welle et al., "EEG and MRI correlates of mild cognitive impairment and Alzheimer's disease," Neurobiology of Aging, vol. 28, no. 9, pp. 1322-1329, 2007.

[7] Z. Y. Jiang, "Study on EEG power and coherence in patients with mild cognitive impairment during working memory task," Journal of Zhejiang University Science B, vol. 6, no. 12, pp. 1213-1219, 2005.

[8] K. van der Hiele, A. A. Vein, C. G. S. Kramer et al., "Memory activation enhances EEG abnormality in mild cognitive impairment," Neurobiology of Aging, vol. 28, no. 1, pp. 85-90, 2007.

[9] D. Adamis, S. Sahu, and A. Treloar, "The utility of EEG in dementia: a clinical perspective," International Journal of Geriatric Psychiatry, vol. 20, no. 11, pp. 1038-1045, 2005.

[10] R. J. Croft and R. J. Barry, "Removal of ocular artifact from the EEG: a review," Neurophysiologie Clinique, vol. 30, no. 1, pp. 5-19, 2000.

[11] D. Hagemann and E. Naumann, "The effects of ocular artifacts on (lateralized) broadband power in the EEG," Clinical Neurophysiology, vol. 112, no. 2, pp. 215-231, 2001.

[12] I. I. Goncharova, D. J. McFarland, T. M. Vaughan, and J. R. Wolpaw, "EMG contamination of EEG: spectral and topographical characteristics," Clinical Neurophysiology, vol. 114, no. 9, pp. 1580-1593, 2003.

[13] R. P. Brenner, R. F. Ulrich, and D. G. Spiker, "Computerized EEG spectral analysis in elderly normal, demented and depressed subjects," Electroencephalography and Clinical Neurophysiology, vol. 64, no. 6, pp. 483-492, 1986.

[14] R. J. Croft and R. J. Barry, "EOG correction of blinks with saccade coefficients: a test and revision of the aligned-artefact average solution," Clinical Neurophysiology, vol. 111, no. 3, pp. 444-451, 2000.

[15] R. J. Croft, J. S. Chandler, R. J. Barry, N. R. Cooper, and A. R. Clarke, "EOG correction: a comparison of four methods," Psychophysiology, vol. 42, no. 1, pp. 16-24, 2005.

[16] T. Gasser, J. C. Schuller, and U. S. Gasser, "Correction of muscle artifacts in the EEG power spectrum," Clinical Neurophysiology, vol. 116, no. 9, pp. 2044-2050, 2005.

[17] T. P. Jung, S. Makeig, M. Westerfield, J. Townsend, E. Courchesne, and T. J. Sejnowski, "Removal of eye activity artifacts from visual event-related potentials in normal and clinical subjects," Clinical Neurophysiology, vol. 111, no. 10, pp. 1745-1758, 2000.

[18] T. P. Jung, S. Makeig, C. Humphries et al., "Removing electroencephalographic artifacts by blind source separation," Psychophysiology, vol. 37, no. 2, pp. 163-178, 2000.
[19] R. C. Petersen, G. E. Smith, S. C. Waring, R. J. Ivnik, E. G. Tangalos, and E. Kokmen, "Mild cognitive impairment: clinical characterization and outcome," Archives of Neurology, vol. 56, no. 3, pp. 303-308, 1999.

[20] G. McKhann, D. Drachman, M. Folstein et al., "Clinical diagnosis of Alzheimer's disease: report of the NINCDSADRDA work group under the auspices of Department of Health and Human Services Task Force on Alzheimer's disease," Neurology, vol. 34, no. 7, pp. 939-944, 1984.

[21] J. I. Skeikh and J. A. Yesavage, "Geriatric Depression Scale (GDS): recent evidence and development of a shorter version," in Clinical Gerontology: A Guide to Assessment and Intervention, T. L. Brink, Ed., pp. 165-173, The Haworth Press, New York, NY, USA, 1986.

[22] D. I. Kaufer, J. L. Cummings, P. Ketchel et al., "Validation of the NPI-Q, a brief clinical form of the Neuropsychiatric Inventory," Journal of Neuropsychiatry and Clinical Neurosciences, vol. 12, no. 2, pp. 233-239, 2000.

[23] P. Aalten, M. E. De Vugt, R. Lousberg et al., "Behavioral problems indementia: a factor analysis of the neuropsychiatric inventory," Dementia and Geriatric Cognitive Disorders, vol. 15, no. 2, pp. 99-105, 2003.

[24] M. Roth, E. Tym, and C. Q. Mountjoy, "CAMDEX. A standardised instrument for the diagnosis of mental disorder in the elderly with special reference to the early detection of dementia," British Journal of Psychiatry, vol. 149, pp. 698-709, 1986.

[25] G. E. Schwartz, P. L. Fair, and P. Salt, "Facial muscle patterning to affective imagery in depressed and nondepressed subjects," Science, vol. 192, no. 4238, pp. 489-491, 1976.

[26] M. M. Bradley, "Emotion and motivation," in Handbook of Psychophysiology, J. Cacioppo, L. Tassinary, and G. Berntson, Eds., pp. 602-643, Cambridge University Press, Cambridge, UK, 2000. 


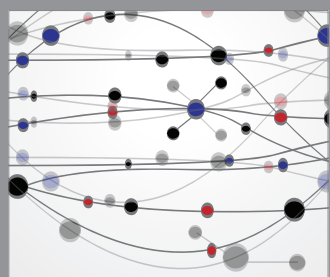

The Scientific World Journal
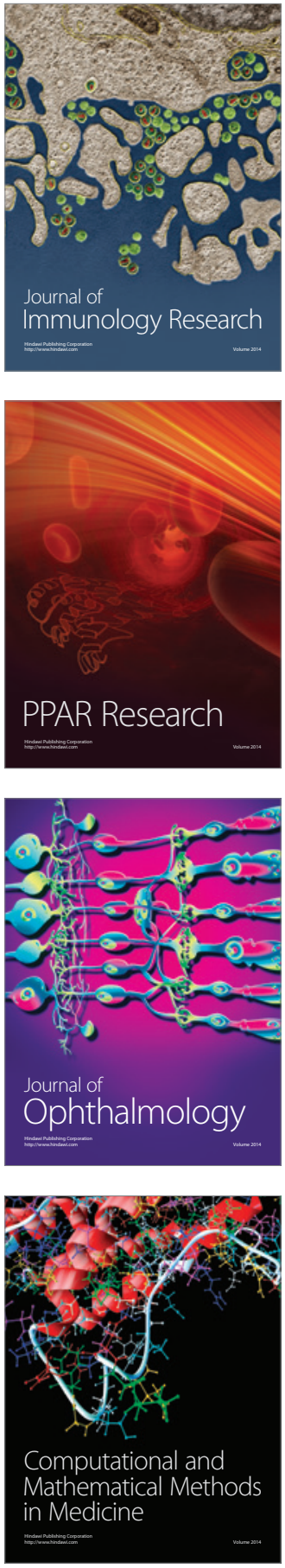

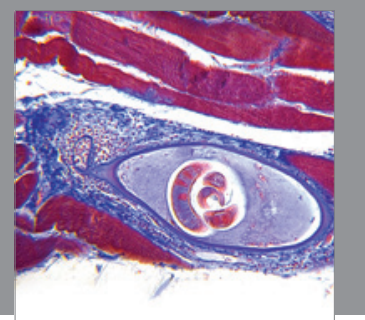

Gastroenterology

Research and Practice
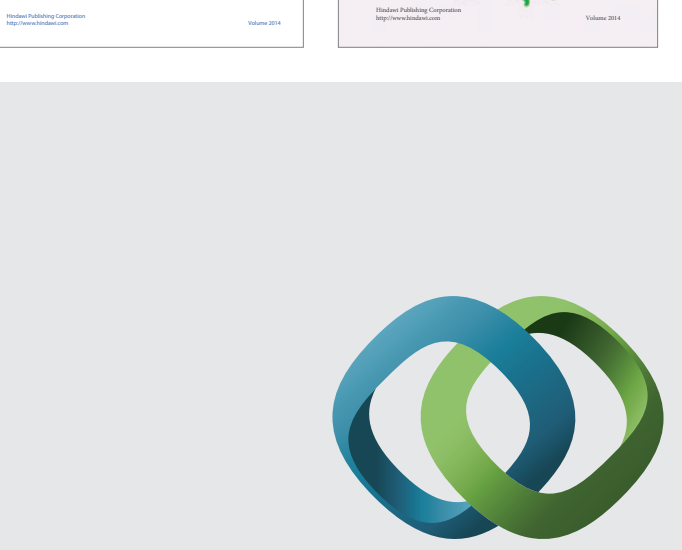

\section{Hindawi}

Submit your manuscripts at

http://www.hindawi.com
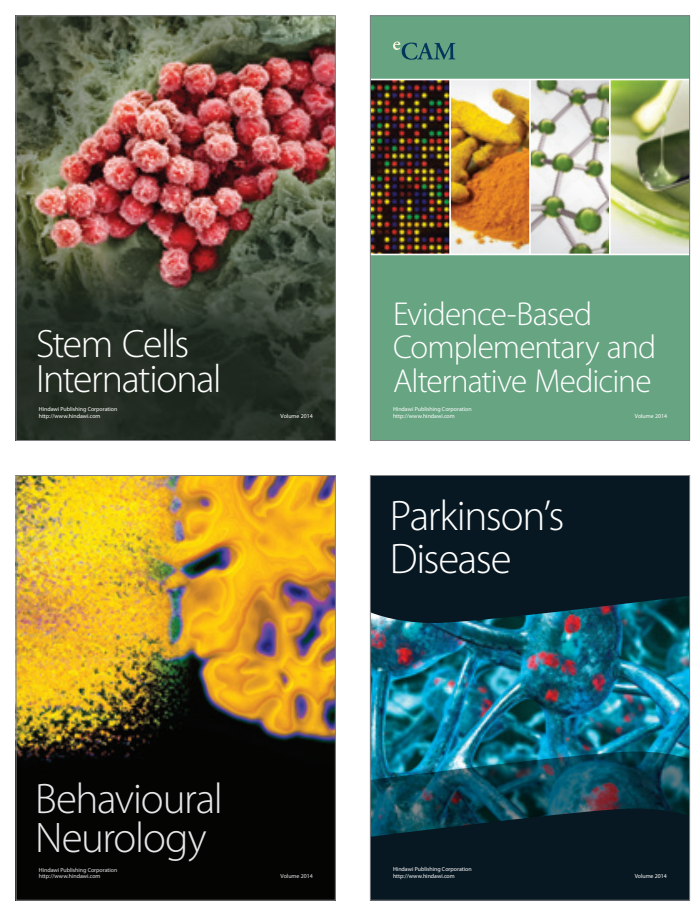

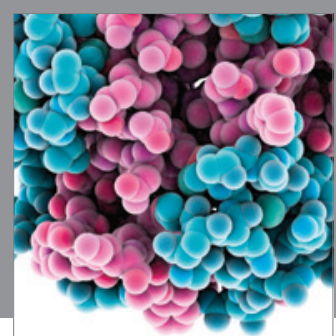

Journal of
Diabetes Research

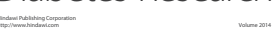

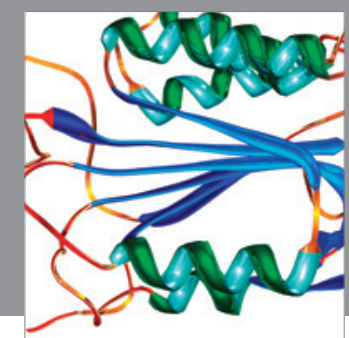

Disease Markers
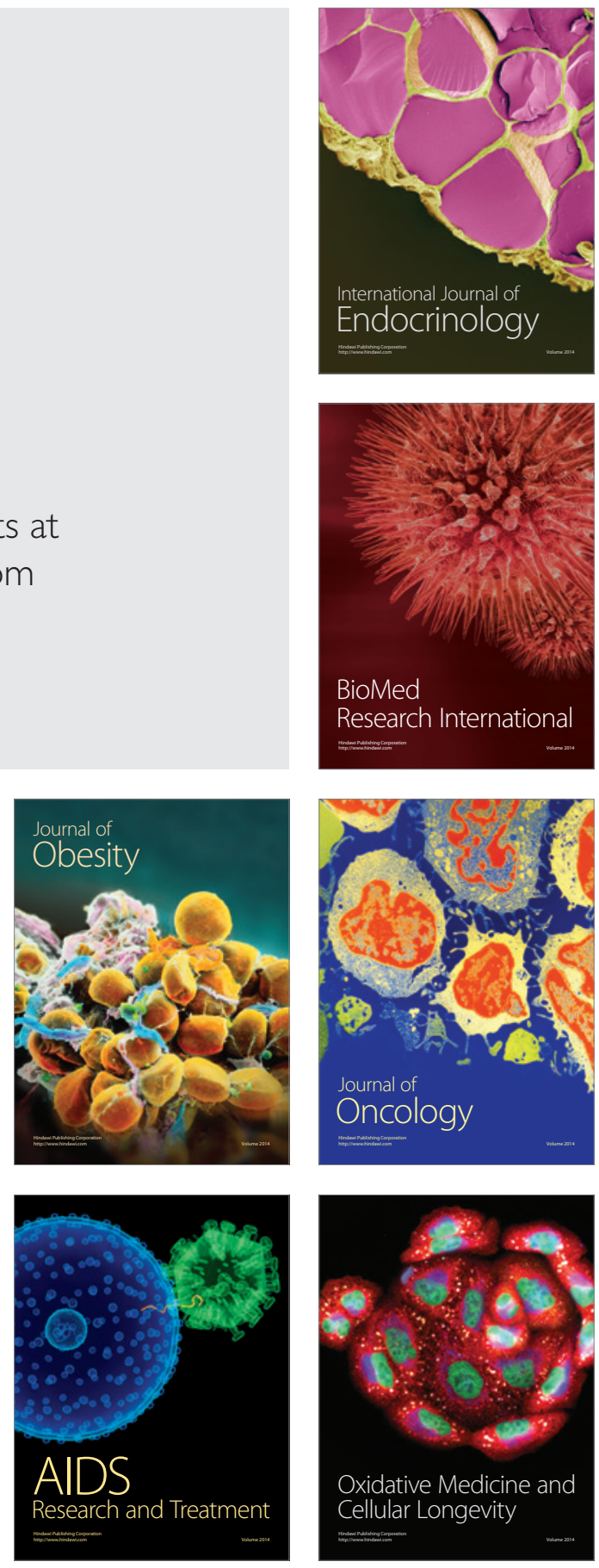